\title{
Revista Brasileira de Enfermagem REBEn Correlação entre resistência carotídea e
autonomia funcional de mulheres idosas
}

\author{
Correlation between the carotid resistance and functional autonomy of old women \\ Correlación entre resisténcia carotídea y autonomía funcional de mujeres ancianas
}

Yúla Pires da Silveira Fontenele de

Meneses

Especialista, Educadora Física da Universidade Estadual do Piauí, Teresina, PI e da Faculdade NOVAFAPI.

Endereço para Contato Universidade Estadual do Piauí (UESPI) Av. João Cabral, s/n, CEP. 64000-000, Teresina, PI, Brasil. yula@globo.com

Patrícia Uchoa Leitão Cabral

Especialista, Educadora Física. Professora da Universidade Estadual do Piauí e da Faculdade NOVAFAPI, Teresina, PI.

Flávia Maria Campos Abreu Fisioterapeuta.

Rodrigo Gomes de Souza Vale

Educador Físico.

Francisca Cecília Viana Rocha

Especialista, Enfermeira, Professora da Faculdade NOVAFAPI, Teresina, PI.

Arméle Dornelas de Andrade

Doutora, Fisioterapeuta, Professora da Universidade Federal de Pernambuco, Recife, $P E$

Submissão: 20/05/2007

Aprovação: 03/07/2007

\section{RESUMO}

Este estudo objetivou correlacionar resistência da artéria carótida e autonomia funcional de idosas. Avaliou-se a resistência da artéria carótida pelo método de ultra-som Doppler e a Autonomia Funcional por testes relacionados a atividades da vida diária (Protocolo GDLAM). Para a descrição dos dados, calculouse média e desvio padrão, em que a resistência da carótida interna direita obteve respectivamente $(0,71$ $\pm 0,07)$ e o Índice geral de Autonomia Funcional apresentou $(30,40 \pm 6,31)$. 0 coeficiente de correlação de Spearman mostrou forte associação entre as variáveis do estudo $(r=0,998 ; p=0,000)$. Os resultados sugerem que quanto maior o índice de resistência da artéria carótida, maior será a dificuldade de execução das tarefas da vida diária.

Descritores: Artéria carótida interna; Atividades cotidianas; Idoso.

\section{ABSTRACT}

This study had as objective to correlate the resistance of the carotid artery and the functional autonomy of old women. The resistance of the carotid artery was evaluated through the method called Doppler and the functional autonomy through tests related to daily activities (Protocol GDLAM). In the description of the data it was used measures of localization and dispersion. The internal right carotid resistance got mean and pattern deviation respectively $(0,71 \pm 0,07)$ and functional autonomy mean and pattern deviation respectively $(30,40 \pm 6,31)$. The coefficient of the correlation of Spearman showed a strong association between the variation of the study $(r=0,998 ; p=0,000)$. The results suggest that the bigger the table of contents of the resistance of the carotid artery is the bigger will be the difficulty of execution of the daily life tasks.

Descriptores: Carotid artery internal; Activities of daily living; Aged.

\section{RESUMEN}

Este estudio objetivou correlacionar resistência de la arteria carótida interna derecha Y autonomía funcional de ancianas. Se evaluó la resistência de la arteria carótida por el método de ultra-sonido Doppler e a autonomia funcional a través de testes relacionados la actividad da vida diaria (Protocolo GDLAM). Em la descripción de los datos, se calculou media y desvío padrón, donde la resistência de la arteria carótida interna derecha tuvo respectivamente $(0,71 \pm 0,07)$ y em lo índice geral de la autonomía funcional fueron respectivamente $(30,40 \pm 6,31)$. Lo coeficiente de la correlación de Spearman mostrou fuerte associación entre las variables de el estudio $(r=0,998 ; p=0,000)$. Los resultados sugerem que cuanto mayor lo índice de la resistência de la arteria carótida, mayor fuerón las dificuldades de execucusión de las actividad de la vida diaria.

Descriptores: Arteria carótida interna; Actividades cotidianas; Anciano.

Meneses YPSF, Cabral PUL, Abreu FMC, Vale RGS, Rocha FCV, Andrade AD. Correlação entre resistência carotídea e autonomia funcional de mulheres idosas. Rev Bras Enferm 2007 jul-ago; 60(4):382-6.

\section{INTRODUÇÃO}

O envelhecimento geralmente está associado a limitações de caráter fisiológico e funcional, sendo a soma de alterações biológicas, psicológicas e sociais que ocorrem com o passar dos anos em qualquer indivíduo(1).

Processos patológicos de aumento da resistência da carótida poderão ser desencadeados durante 0 envelhecimento humano, podendo estar relacionados a vários fatores como alimentação, hábitos de vida, provocadores de estenose ou aterosclerose do sistema arterial cerebral(2).

No processo de envelhecimento a resistência da carótida é aumentada, sendo fato patológico relacionado 
a vários fatores inclusive aos hábitos de vida ${ }^{(3)}$. É através da artéria carótida que o coração irriga o cérebro; estando congestionada, esta via reduz 0 aporte de oxigênio ao cérebro(3,4).

As carótidas são em número de duas, com origem no tórax (arco aórtico), que passam lateralmente pelo pescoço em lados opostos até o crânio, cada uma irrigando o seu lado craniano, havendo, entretanto, comunicação entre as mesmas na caixa craniana ${ }^{(4,2)}$.

A placa de ateroma é um processo degenerativo localizado na camada intima dos vasos, caracterizado por acúmulo de lípides plasmáticos, fibras do tecido conjuntivo e células locais e circulantes ${ }^{(3)}$. A formação destas placas leva a um estreitamento da luz do vaso e à redução do fluxo, caracterizando-se como estenose da carótida ou ateroma ${ }^{(2,4,5)}$. $O$ ateroma reduz o fluxo sanguíneo cerebrale aumenta a resistência da artéria em torno de $15 \%$ em homens e $10 \%$ em mulheres a cada dez anos de vida ${ }^{(4,6)}$.

Referente ao controle da resistência carotídea, estudos afirmam que índices de resistividade carotídea têm involuído, através de intervenção hormonal ${ }^{(2,4,5)}$, quando comparado a grupos de idosas que sofreram apenas intervenção de cinesioterapia aeróbica. Os autores atribuem este resultado ao pouco tempo de intervenção, não proporcionando modificações significativas, ou a intensidade do exercício ter sido insuficiente ${ }^{(5)}$.

A diminuição da capacidade de desempenho físico durante a vida é freqüentemente considerada mais como uma conseqüência das condições de trabalho e dos hábitos de vida do que de incapacidade biológica. Isto pode comprometer a autonomia funcional do indivíduo quando envelhecetornando as pessoas dependentes de outras ${ }^{(7,8)}$.

As modificações fisiológicas acometidas, principalmente nas mulheres, após a idade adulta, influenciam diretamente em todo o seu desempenho social e racional, podendo ser amenizadas com a prática de atividade física não aleatória(5).

A inatividade física combinada com o envelhecimento biológico poderá resultar em grandes perdas nas capacidades funcionais do indivíduo idoso ${ }^{(8)}$. Mesmo mantendo atividade física constante, com o passar dos anos, podem ser detectadas perdas na força dinâmica, vindo a comprometer a execução de atividades de vida diária (AVDs) ${ }^{(8,9)}$.

As alterações funcionais as quais os idosos são acometidos com 0 passar dos anos, associadas às doenças crônicas, podem levá-los a deteriorização da habilidade de manutenção da independência, desencadeando modificações em toda a estrutura física e funcional do indivíduo e, de um modo especial, no sistema músculo-esquelético, proporcionando graus consideráveis de perda na autonomia funcional dos indivíduos $\mathrm{s}^{(9,10)}$.

Coma idade a resistência muscular é geralmente mais bem preservada do que a força muscular máxima, embora alguns autores ${ }^{(6,9,11)}$ afirmem também ter redução na resistência aeróbica. A perda de força estáligada diretamenteà atrofia muscular que ocorre devido ao decréscimo tanto no tamanho das fibras musculares quanto no número. Aesta perda de força e atrofia muscular também é associada a denervação seletiva de fibras musculares, sendo as maiores perdas funcionais entre as unidades motoras maiores e mais rápidas ${ }^{(8,10)}$.

Poucos são os estudos sobre a influência da resistência da artéria carótida sobre a autonomia funcional de idosos, contudo, redução nos níveis de resistência carotídea pode implicar em aumento no fluxo sanguíneo cerebral, reduzindo fatores de risco para doenças cerebrovasculares, paralelo a melhorias na autonomia funcional de mulheres idosas ${ }^{(12)}$.

Sendo assim, o objetivo deste estudo foi correlacionar o nível de associação entre a resistência da artéria carótida interna direita com a autonomia funcional de mulheres idosas, observando a oclusão nas placas do bulbo, a utilização de medicamentos e as patologias existentes.

\section{METODOLOGIA}

O presente estudo caracteriza-se por uma metodologia quantitativa com uma amostra de conveniência, desde que atendesse aos critérios de inclusão da pesquisa.
A amostra é formada por 27 mulheres, na faixa etária entre 60 e 78 anos de idade, aparentemente saudáveis (sem apresentar queixa de patologias no momento do estudo) e sedentárias, pelo menos há três meses, oriundas de projetos sociais de igreja, desenvolvidos em bairros da periferia na cidade de Teresina, no Estado do Piauí.

Os indivíduos, após participarem de uma palestra de apresentação do projeto-pesquisa, passaram por avaliação médica e física e assinaram um termo de consentimento livre e esclarecido. 0 critério de inclusão estabelecia que os indivíduos deveriam ser do sexo feminino, ter idade entre 60 e 78 anos e conseguir realizar os testes de autonomia funcional. Como critério de exclusão foi considerado toda e qualquer condição aguda ou crônica que fosse fator de impedimento para a realização dos testes.

Este estudo foi aprovado pelo Comitê de Ética da Faculdade de Ciências Médicas da Universidade Estadual do Piauí, de acordo com as normas da Resolução 196/96, do Conselho Nacional de Saúde, sobre pesquisa que envolve seres humanos.

Para avaliar a autonomia funcional, utilizou-se o protocolo do Grupo de Desenvolvimento Latino-Americano para a Maturidade (GDLAM) $)^{(13,14)}$, que consiste na realização de cinco testes, caracterizados por quesitos necessários à avaliação da autonomia funcional no desempenho das atividades físicas da vida diária (AVD) $)^{(8)}$. Teste: C10M (caminhar 10 metros) $)^{(15)}$, LPS (levantar da posição sentada) $)^{(11)}$, LCLC (levantar-se da cadeira e locomover-se pela casa) ${ }^{(8)}$, LPDV (levantar-se da posição de decúbito ventral) $)^{(10)}$, VTC (vestir e tirar a camisa) ${ }^{(13)}$.

Os instrumentos de avaliação utilizados nos testes do Protocolo GDLAM foram: cronômetros, cadeira de 45 centímetros de altura, do assento ao solo, 2 colchonetes, 2 cones, trena de 7 metros, camiseta GG.

As idosas também foram submetidas a Doppler de carótida para mensuração do índice de resistividade em centímetros/segundo, utilizandose 0 aparelho de ultra-som de alta resolução com Doppler com Transdutor Linear de 7,5Mz, da marca MEDSON, modelo SONOACE 8000.

O tratamento estatístico foi realizado no programa SPSS 14.0. Os dados das características são apresentados por média e desvio-padrão. Os quartis foram utilizados para a variável resistência da carótida. Para a inferência estatística, utilizou-se o teste de Shapiro-Wilk para verificar a normalidade da amostra. Foi usado o teste de correlação de Spearman para analisar o nível de associação entre as variáveis da pesquisa. 0 estudo admitiu o valor de $p<0,05$ para a significância estatística.

\section{RESULTADOS}

Os dados da tabela 1 apresentam a descrição da amostra e sua classificação conforme o padrão GDLAM.

De acordo com o IR.CID encontrado, $25 \%$ das idosas fazem parte do primeiro quartil, onde $Q_{1}=0,66$. O segundo quartil correspondeu a $Q_{2}=0,70$ e o $Q_{3}=0,73$, sabendo-se que neste último, os valores chegaram a 0,86 , identificando riscos à saúde, porém, não as impossibilitando da prática das atividades da vida diária.

Os dados relatados foram classificados segundo o índice geral de autonomia funcional GDLAM (IG) e representados segundo tabela de classificação específica $^{(14)}$.

Através do teste de Spearman, observou-se que o IRCID apresentou um coeficiente de correlação significativa com a bateria de testes e IG do protocolo de avaliação da autonomia funcional GDLAM.

\section{DISCUSSÃO}

O índice de resistividade da carótida interna direita mostrou uma correlação positiva com todos os testes do protocolo de avaliação da autonomia funcional, ou seja, quanto maior o IRCID maior a dificuldade de realizar a bateria de testes de autonomia funcional do protocolo GDLAM.

Foram calculados os quartis do índice de resistividade da artéria carótida 
Tabela 1. Características da amostra referente à autonomia funcional de idosas

\begin{tabular}{lcccc}
\hline Variáveis & Média & DP & Valor-p (SW) & $\begin{array}{c}\text { Classificação } \\
\text { GDLAM }\end{array}$ \\
\hline Idade & 68,67 & 4,52 & 0,676 & - \\
LPS & 11,36 & 2,39 & 0,339 & Fraco \\
LCLC & 46,70 & 16,82 & 0,000 & Fraco \\
VTC & 13,08 & 2,97 & 0,120 & Regular \\
C10m & 7,83 & 1,08 & 0,857 & Fraco \\
LPDV & 5,17 & 2,25 & 0,009 & Fraco \\
IG & 30,40 & 6,31 & 0,000 & Fraco \\
IR.CID & 0,71 & 0,07 & 0,291 & - \\
\hline
\end{tabular}

LPS = levantar da posição sentada; LCLC = levantar da cadeira e locomover-se pela casa. VTR = vestir e tirar a camiseta; $\mathrm{C} 10 \mathrm{~m}=$ caminhar 10 metros; $\mathrm{LPDV}=$ levantar da posição de decúbito ventral; IG = índice geral do protocolo de avaliação de GDLAM; IR.CID = Indice de resistividade da carótida interna direita (centímetros/segundo). Média = tempo (segundos); DP = desvio padrão; SW = Shapiro-Wilk.

Tabela 2. Correlação entre autonomia funcional e resistência da carótida de mulheres idosas

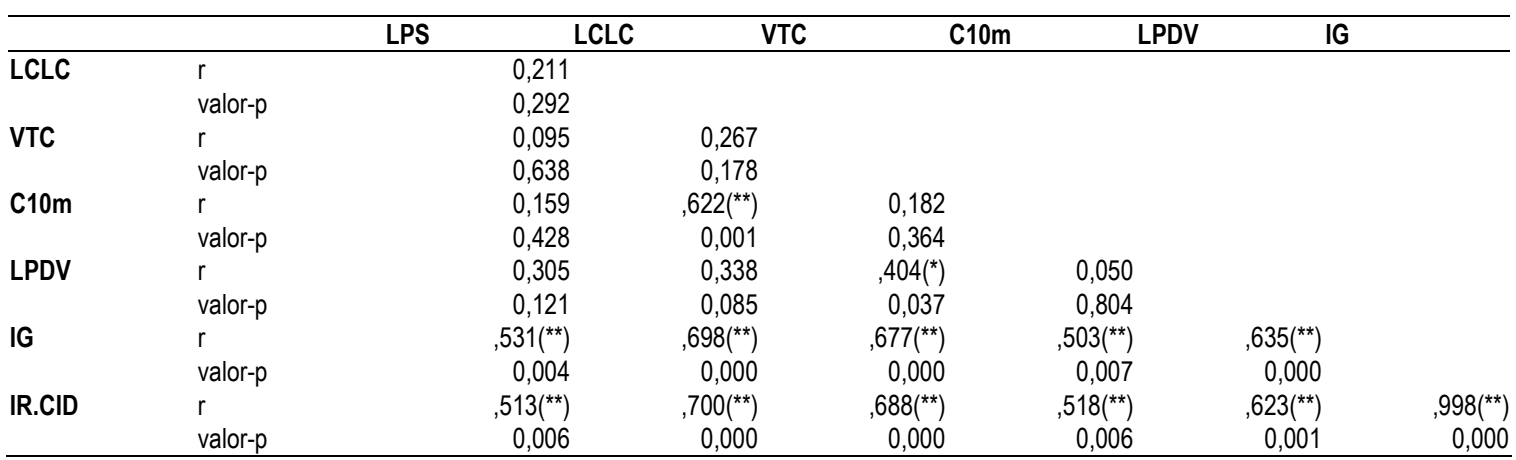

C10m = caminhar 10 metros; LPS = levantar da posição sentada; LPDV = levantar da posição de decúbito ventral; LCLC = levantar da cadeira e locomover-se pela casa Média=tempo (segundos); IR.CID = Índice de resistividade da carótida interna direita.

${ }^{* *} p<0,01$;

${ }^{*} p<0,05$

Tabela 3. Valores médios de autonomia funcional de idosas em pesquisas diversas.

\begin{tabular}{lllll}
\hline DLAM & C10M & LPS & LPDV & LCLC \\
\hline Vale, 2005(23) & 6,56 & 11,15 & 4,47 & 45,03 \\
Abreu, 2005(5) & 6,98 & 10,40 & 3,11 & 38,11 \\
Vale, 2006(11) & 6,31 & 10,38 & 3,32 & - \\
Meneses, 2007 & 7,83 & 11,36 & 5,17 & 46,6 \\
\hline
\end{tabular}

C10m = caminhar 10 metros; LPS = levantar da posição sentada; LPDV = levantar da posição de decúbito ventral; LCLC = levantar da cadeira e locomover-se pela casa. Média=tempo (segundos).

interna direita, por apresentar uma variabilidade considerável.

As médias encontradas, foram superiores ao que se tem de registro em pesquisas ${ }^{(13,16)}$ sobre IR.CID, com médias entre $0,64 \mathrm{~cm} / \mathrm{s}$ e $0,70 \mathrm{~cm} / \mathrm{s}$ para não fumantes e fumantes, respectivamente, de populações diferentes.

Placas de ateroma, com obstruções entre 20 a $37 \%$, hipertensão arterial e um caso em que os dois fatores estão associados a diabetes foram detectados em idosas desta pesquisa; contudo, não foram excluídas deste estudo por estarem sob controle medicamentoso e o exame médico não as ter colocado em situação de risco. Porém, encontravam-se fora da normalidade nos testes estatísticos, podendo ter contribuído diretamente para o aumento da resistência da carótida, comprometendo o fluxo sanguíneo cerebral e a autonomia funcional.

Corroborando com esta pesquisa, Galetta et al(4), mostraram que sujeitos idosos sedentários apresentaram maior rigidez da camada medial da artéria carótida, assim como menor vasodilatação dependente de endotélio, quando comparados com atletas idosos. Relacionando o tempo de atividade física com a rigidez arterial, através de Doppler de carótida, foi detectado que, quanto maior o tempo de atividade física nos momentos de lazer, menor 0 nível de rigidez arterial ${ }^{(2)}$.

Maior rigidez arterial pode ser um preditor de aterosclerose nas artérias coronárias, vindo a prejudicar o desempenho nas atividades da vida diária e reduzir a expectativa de vida ${ }^{(10,11)}$. Os valores encontrados estão dentro de índices aceitáveis para a manutenção das atividades da vida diária.

Foi observada redução significativa da resistência da artéria carótida direita, em diferentes métodos de intervenção hormonal, obtendo melhorias não só com a prática de atividade física cardiorrespiratória, em intensidade moderada, mas também com o uso de hormônios ${ }^{(5,16)}$.

Estudos sobre redução no IRCID são escassos, por isso se tornam importantes, principalmente por provocarem discussões a respeito do uso da terapia hormonal e prescrições de atividades físicas neuromusculares e 
cardiorrespiratórias ${ }^{(5)}$.

Ganhos na diminuição no IRCID, indicam um possível aumento do fluxo sangüíneo cerebral, sendo um retardo dos fatores de risco para doenças cerebrovasculares, de memória e cogniçãa ${ }^{(3,5,17)}$.

Cognição prejudicada na senescência pode ser restabelecida através de atividades físicas persistentes e reposições hormonais que melhorem o estado funcional, a circulação sangüínea cardiovascular e cerebral(4,15,17); 0 exercício físico, quando praticado desde idades mais jovens, poderá retardar 0 aparecimento de patologias ou limitações na vida do idoso ${ }^{(10,18)}$.

Através da média adquirida no protocolo de avaliação da autonomia funcional GDLAM, comparou-se os resultados desta pesquisa a outras pesquisas, como mostra a Tabela 3.

A autonomia funcional (GDLAM) encontrada nesta pesquisa quando comparada a três grupos diferentes de idosas sedentárias, no município de Araruama-RJ(13), em Barbacena-MG ${ }^{(5)}$, e na região dos lagos - $R J^{(9)}$, observaram-se índices de sedentarismo inferiores. O que poderia ser justificado por fatores como condição sócio-econômica, altos níveis de sedentarismo na prática de atividades da vida diária, dentre outros.

Fatores determinantes da autonomia funcional em idosos, concluem que a renda per capita e o grau de escolaridade não estão associados ao nível de dependência, os quais podem influenciar em idades mais avançadas ${ }^{(19)}$. No entanto, com respeito ao modo de vida, morar só, foi tido como fator protetor contra a dependência; e maior probabilidade de declínio da autonomia funcional, a redução gradativa das relações sociais como, fazer visitas e ir à igreja, pois deixam os indivíduos mais ativos, capazes de manter por mais tempo a autonomia funcional(19).

A amostra deste estudo foi constituída por idosas de baixa renda, que se utiliza de transporte público para deslocamento, moram na periferia da cidade, participam de grupos sociais de pastorais da igreja católica. Durante avaliação médica foi detectado o uso de medicamentos que controlam algumas patologias como hipertensão, diabetes, sobrepeso e hipercolesterolemia, estes achados podem justificar o fato de que apesar de terem maior atividade para realização das tarefas da vida diária, não possuírem domésticas ou meio de transporte particular, não se pode descartar um trabalho de orientação para as modificações fisiológicas advindas no processo de envelhecimento.

Condições de vida e de saúde de idosos de Florianópolis ${ }^{(20)}$, foram avaliadas através do Questionário Internacional de Atividades Físicas, desenhando o perfil de idosos e surpreendendo pesquisadores ao detectar saúde entre boa e ótima em $73 \%$ dos entrevistados. Aavaliação das atividades da vida diária e da saúde mental demonstra que os mais ativos são mais independentes e têm menos indicadores negativos de saúde mental.

Uma limitação de nosso estudo foi a heterogeneidade da nossa amostra sobre a autonomia funcional das idosas de Teresina, observado no teste de normalidade de Shapiro-Wilk. No entanto, este fato não invalida nossos achados uma vez que na literatura é comum esta variabilidade de autonomia funcional nesta faixa etária.

Estudo ${ }^{(4)}$, também com amostra heterogênea, detectou que mulheres desmotivadas para o trabalho, mesmo com hipertensão e vida social limitada, foram associadas a dependências leves em avaliação da autonomia funcional.

Analisando o gráfico de correlação do IRCID e AF nesta pesquisa, sugere-se que quanto maior o IRCID maior foi a dificuldade em realizar os testes que envolviam grandes grupos musculares e mudanças de direção.

\section{CONCLUSÃO}

A correlação entre o IRCID e a autonomia funcional de mulheres idosas foi positiva, mostrando que quanto maior for o IRCID maior foi a dificuldade de realizar os testes de autonomia funcional do protocolo de GDLAM.

A análise direta do IR. CID da amostra possibilitou traçar objetivos para posteriores pesquisas que preconizem a prática regular da atividade física para manutenção das atividades da vida diária e prevenção de patologias cerebrovasculares, contribuindo para a melhoria da autonomia funcional em mulheres idosas.

O perfil das resistências das artérias carótidas internas é um método proposto para estudos futuros que possam adequar-se à prevenção de doenças cerebrovasculares causadas pelo envelhecimento normal, manutenção da cognição, memória e fluxo cerebral, através de medidas terapêuticas ou preventivas como treinamento físico variado.

\section{REFERÊNCIAS}

1. Zago AS, Gobbi S. Comparativos da aptidão funcional de mulheres entre 60 e 70 anos. R Bras Ciencia Movimento 2003 jun;11(2): 77-86.

2. Schmidt-Trucksãss A, Grathwohl D, Frey I, Schmid A, Boragk $\mathrm{R}$, Upmeir $\mathrm{C}$, et al. Relation of leisure-time physical activity to structural and functional arterial properties of the common carotid artery in male subjects. Atherosclerosis 1999;145(1):107-14.

3. Kotsis VT, Pitiriga VCh, Stabouli SV, Papamichael CM, Toumanidis ST,Rokas SG, Zakopoulos NA. Carotid artery intima media thickness could predict the presence of coronary artery lesions. Am J Hipertension 2005;18(5):601-6.

4. Galetta F, Franzoni F, Virdis A, Ghiadoni L,Taddei S, Santoro G. Endotelium-dependent vasodilation and carotid artery wall remodeling in athletes and sedentary subjects. Atherosclerosis 2006;186(1):184-92.

5. Abreu FMC. Fisioterapia aplicada à geriatria e gerontologia. Fitness \& Performance J 2003;2(6):312-15.

6. Amorim FS, Dantas EHM. Efeitos do treinamento da capacidade aeróbica sobre a qualidade de vida e autonomia de idosos. Fitness e Performance J 2002;1(3):47-59.

7. Rosa TEC, Benício MHD'A, Latorre MRDO, Ramos LR. Fatores determinantes da capacidade funcional entre idosos. Rev Saúde Pública 2003 37(1)

8. Andreotti RA, Okuma SS. Validação de uma bateria de testes de atividades da vida diária para idosos fisiocamente dependentes. Rev Paul Educação Física 1999;13(1):46-66
9. Vale RGS, Barreto ACG, Novaes JS, Dantas EHM. Efeitos do treinamento resistido na força máxima, na flexibilidade e na autonomia funcional de mulheres idosas. Rev Bras Cineantropom Desempenho Hum 2006;8(4):52-8.

10. Alexander NB. Rising from the floors in older adults. $J$ Am Geriatrics Society. 1997; 45(5): 564-69 20.

11. Guralnik JM. Lower-extremity function in persons over de age of 70 years as a predictor of subsequent disability. New England $\mathrm{J}$ Med 1995;332(9):556-61.

12. Freitas EV, Py L, Néri AL, Caçado FAX, Goerzoni ML, Rocha SM. Tratado de Geriatria e Gerontologia. Rio de Janeiro (RJ): Guanabara Koogan; 2002.

13. Vale RGS, Novaes JS, Dantas, EHM. Efeitos do treinamento de força e de flexibilidade sobre a autonomia de mulheres senescentes. R Bras Ciência e Movimento 2005;13(2):33-40.

14. Dantas EHM, Vale RGS. Protocolo GDLAM de Avaliação da Autonomia Funcional. Fitness \& Performance J 2004;3(3):169-180.

15. Sipilã S, Multanen J, Kallinen M, Era P, Suominen H. Effects of strength and endurance training on isometric muscle strength and walking speed in elderly women. Acta Physiol Scand 1996;156:457-64

16. Massabki JOP. Terapia hormonal na menopausa em mulheres fumantes e não fumantes: estudo vascular. Rev Ginecol Obstet 2001;12(3):121-25.

17. Matsudo SM. Envelhecimento Atividade Física e Saúde. Rev Min Educ Fís 2002;10(1):193-207. 
Meneses YPSF, et al.

18. Monteiro MI, Fernandes ACP. Capacidade de trabalho de trabalhadores de empresa de tecnologia da informação. Rev Bras Enferm 2006;59(5):603-8.

19. Silva DK, Nahas MV. Atividade física habitual e qualidade de vida relacionada à saúde em mulheres com doença vascular periférica. Rev Bras Ciência e Movimento 2004;12(4):63-8.

20. Kington RS, Smith JP. Socioeconomic Status and racial and ethnic differences in functional status associated with chronic diseases. Am J Public Health 1997;87:805-10. 